\title{
AVALIAÇÃO DE UMA TÉCNICA PARA GERAÇÃO DE MODELOS DIGITAIS DE SUPERFÍCIE UTILIZANDO MÚLTIPLAS IMAGENS
}

Assessment of a technique for Digital Surface Model generation using multi images

\author{
RAQUEL ALVES DE OLIVEIRA \\ ANTONIO MARIA GARCIA TOMMASELLI
}

\author{
Programa de Pós-Graduação em Ciências Cartográficas \\ Faculdade de Ciências e Tecnologia - Departamento de Cartografia \\ UNESP - Universidade Estadual Paulista \\ Rua Roberto Simonsen, 305, 19060-900 Presidente Prudente, SP. \\ raquel88@gmail.com, tomaseli@fct.unesp.br
}

\begin{abstract}
RESUMO
A utilização de imagens ópticas para a geração de Modelos Digitais de Superfície (MDSs) é explorada há muitos anos e os resultados dependem, além das características do projeto (resolução das imagens, tamanho da área de sobreposição entre as imagens, etc), das técnicas de correspondência de imagens aplicadas e da capacidade computacional para o processamento das imagens. Os pontos gerados com erros no processo de correspondência influenciam diretamente a qualidade dos produtos obtidos e, consequentemente, aumentam a necessidade da etapa onerosa de edição. O objetivo deste trabalho é a avaliação da técnica de geração de MDSs utilizando a correspondência simultânea entre múltiplas imagens pelo método de busca em linhas verticais (Vertical Line Locus-VLL). Nos experimentos foram utilizadas seis imagens da área urbana de Presidente Prudente-SP, com elemento de resolução aproximado de $7 \mathrm{~cm}$. Para análise da qualidade foram gerados MDSs de uma pequena área contendo áreas homogêneas, padrão repetitivo, objetos em movimento incluindo sombras e árvores grandes. O MDS obtido foi comparado com dados de varredura a LASER (Light Amplification by Simulated Emission of Radiation) e com o MDS gerado pelo software Leica Photogrammetric Suite (LPS). Os resultados mostraram a compatibilidade da qualidade entre os modelos gerados pela técnica implementada e os dados de referência.
\end{abstract}

Palavras-chave: Modelo Digital de Superfície; Correspondência de Imagens; Busca em Linha Vertical. 


\section{ABSTRACT}

The efficient generation of digital surface model (DSM) from optical images has been explored for many years and the results are dependent on the project characteristics (image resolution, size of overlap between images, among others), of the image matching techniques and the computer capabilities for the image processing. The points generated from image matching have a direct impact on the quality of the DSM and, consequently, influence the need for the costly step of edition. This work aims at assessing experimentally a technique for DSM generation by matching of multiple images (two or more) simultaneously using the vertical line locus method (VLL). The experiments were performed with six images of the urban area of Presidente Prudente/SP, with a ground sample distance (GSD) of approximately $7 \mathrm{~cm}$. DSMs of a small area with homogeneous texture, repetitive pattern, moving objects including shadows and trees were generated to assess the quality of the developed procedure. This obtained DSM was compared to cloud points acquired by LASER (Light Amplification by Simulated Emission of Radiation) scanning as wells as with a DSM generated by Leica Photogrammetric Suite (LPS) software. The accomplished results showed that the MDS generated by the implemented technique has a geometric quality compatible with the reference models.

Keywords: Digital Surface Models; Image Matching; Vertical Line Locus.

\section{INTRODUÇÃO}

Uma representação tridimensional digital da superfície topográfica é conhecida como Modelo Digital de Terreno (MDT) ou Digital Terrain Model (DTM). Outra denominação correlata encontrada na literatura é Modelo Digital de Superfície (MDS). Este termo, entretanto, se refere à representação que compreende pontos do terreno e acima deste, tais como feições antrópicas (edificações, rodovias) e vegetação.

Os MDSs e MDTs são essenciais para várias aplicações em mapeamento, avaliação de impactos ambientais, políticas públicas para projetos de infraestrutura, geração de ortoimagens e como fonte de dados para Sistemas de Informações Geográficas (SIG). As fontes de dados para a geração de um MDT podem ser curvas de nível digitalizadas de produtos cartográficos pré-existentes, levantamentos realizados em campo, métodos fotogramétricos aplicados sobre imagens aéreas ou em imagens de satélite, dados provenientes de RADAR (RAdio Detection And Ranging) e dados de varredura a LASER (Light Amplification by Simulated Emission of Radiation).

A praticidade e qualidade dos levantamentos utilizando a tecnologia LASER concorrem com as técnicas fotogramétricas, ambas apresentando vantagens e desvantagens para geração de MDS. O levantamento utilizando um sistema de varredura a LASER permite maior facilidade na aquisição de pontos em áreas de vegetação e em áreas homogêneas, que são áreas difíceis de serem corretamente 
estimadas com técnicas de correspondência de imagens. No entanto, o custo deste sistema é relativamente alto comparado com a utilização de Fotogrametria, que tem como vantagem adicional o fornecimento de informações de textura e de radiometria dos elementos presentes na cena.

A geração automática de MDSs utilizando imagens ópticas tem como base essencial o processo de correspondência entre imagens. Embora os algoritmos e as estratégias de correspondência utilizadas, em geral, possam ser diferentes, a precisão e os problemas encontrados são muito similares na maioria das aplicações comerciais ou acadêmicas. Atualmente, as pesquisas na área acadêmica e no setor comercial, foram intensificadas para a geração de MDSs utilizando imagens ópticas (HAALA, 2009) recorrendo-se a uma cobertura com maior número de imagens e maiores superposições.

Os custos adicionais com o aumento na superposição entre as imagens são reduzidos, quando se utilizam câmaras digitais. Além disso, encontram-se no mercado câmaras digitais a um custo relativamente baixo (RUY e TOMMASELLI, 2004), e com alta qualidade radiométrica, que é extremamente favorável para o problema da correspondência de imagens, especialmente em superfícies homogêneas (ROTHERMEL e HAALA, 2011). A ampliação da superposição entre as imagens aumenta o número de observações para um mesmo ponto, beneficiando a qualidade da correspondência e a utilização de métodos que consideram a correspondência simultânea entre múltiplas imagens, como o método de busca em linha vertical (Vertical Line Locus - VLL). Este método pode ser expandido para a utilização de mais do que duas imagens e é amplamente utilizado na área comercial e acadêmica (ZHANG, 2005; IDOETA, 2007; SILVEIRA et al., 2007).

Neste contexto, é de grande relevância investigar o potencial e desenvolver estratégias para a geração de MDSs a partir da abordagem de correspondência densa utilizando múltiplas imagens de câmaras digitais de formato médio.

\section{CORRESPONDÊNCIA DE IMAGENS}

A correspondência de imagens (image matching) é uma das principais técnicas utilizadas em Fotogrametria Digital (SCHENK, 1999; GRUEN, 1985). Esta técnica trata da identificação e medida de pontos homólogos (pontos comuns) em duas ou mais imagens com superposição adequada. Em Fotogrametria as principais aplicações de correspondência de imagens são a geração de MDSs, a medição de pontos fotogramétricos e pontos de controle para orientação de imagens, para o controle de qualidade industrial (pontos com ou sem alvos) e extração automática de feições (GRUEN, 2012). Além disso, é uma técnica também aplicada nas áreas de visão computacional, navegação, vigilância, orientação automática, robótica e análises de imagens médicas (GRUEN, 1985).

Os principais métodos de correspondência de imagens podem ser classificados como métodos baseados em áreas e métodos baseados em feições ou características (Feature-Based Matching - FBM). 
Nos métodos baseados em áreas, o processo de correspondência parte de duas imagens digitais, nas quais são delimitadas as janelas de referência na imagem da esquerda, que são comparadas com janelas na imagem da direita (imagens de busca). Durante este processo compara-se a distribuição dos tons de cinza (ou as bandas espectrais) entre a janela de referência e a janela de busca, delimitada na imagem da direita, utilizando uma função de similaridade, que identifica os pontos homólogos, como a função erro, correlação cruzada, coeficiente de correlação ou pelo método dos mínimos quadrados (MMQ) (HEIPKE, 1996; SCHENK, 1999; GRUEN, 2012). O MMQ é aplicado após ter sido obtida uma solução inicial por uma função de similaridade qualquer e proporciona um resultado com precisão subpixel. Nos métodos baseados em feições a correspondência é realizada pela comparação entre feições extraídas nas imagens. As feições possuem grupos de atributos, como coordenadas, gradientes, tamanho e brilho médio de regiões, comprimento, curvatura, entre outros. Segundo Gruen (2012) este método pode ser dividido em duas etapas: extração de feições (pontos, cantos, segmentos de linhas, bordas, regiões) e em seguida a busca por correspondência entre os conjuntos de feições extraídas. Em geral, as feições podem ser consideradas mais robustas do que níveis de intensidade.

Os principais problemas enfrentados na aplicação de algoritmos de correspondência são a presença de áreas com pouca textura ou de padrões repetitivos, nas quais há várias possibilidades de correspondência; áreas de oclusão; áreas de descontinuidade, que representam as bordas dos objetos da cena, onde há mudanças bruscas de profundidade. Por outro lado, ruídos, mudanças de iluminação e das propriedades refletivas da cena entre as imagens provocam diferenças nos níveis radiométricos digitais. Além disso, existem as distorções geométricas relacionadas aos parâmetros do sistema óptico do sensor, à orientação e ao relevo. As possíveis soluções para diminuir o impacto destes problemas são a combinação de técnicas, utilizando as vantagens de cada uma, o uso de injunções geométricas, a restrição do espaço de busca e a garantia de convergência da função, utilizando técnicas como a busca hierárquica, reamostragem epipolar, busca em linha vertical, entre outras.

\section{VERTICAL LINE LOCUS (VLL)}

O método de busca em linha vertical (Vertical Line Locus - VLL) proporciona uma restrição geométrica no espaço de busca durante o processo de correspondência entre imagens, partindo do espaço objeto para o espaço imagem ao longo de uma linha vertical projetada sobre um ponto no terreno. Desta forma, a correspondência é realizada pelo cálculo da similaridade entre duas ou mais janelas de mesma dimensão delimitadas com centro no ponto do terreno (Figura 1) projetado para as imagens. Em contraste com outros métodos, as posições das janelas de correspondência são alteradas nas duas imagens e movendo-se radialmente em relação ao ponto principal. 
No método VLL é necessário que os parâmetros de orientação exterior (POE) de cada imagem sejam conhecidos e que se tenha a altitude aproximada dos pontos da área de interesse, podendo ser considerados valores aproximados ou médios da altitude da região. Esta aproximação terá influência no tamanho do intervalo de busca na vertical, onde valores mais acurados possibilitam um intervalo de busca menor. A sequência da técnica pode ser descrita formalmente da seguinte maneira: seja um ponto $\mathrm{P}$ com posição planimétrica $(\mathrm{X}, \mathrm{Y})$ conhecida e com altitude aproximada $Z_{0}$. Inicia-se o processo estabelecendo um intervalo de busca na vertical em torno da altitude $Z_{0}$, considerando um limite mínimo (Zmin $=Z_{0}-\Delta Z$ min) e máximo (Zmax $\left.=Z_{0}+\Delta Z \max \right)$ e um valor de incremento $(d Z)$ para a variação de altitude na linha vertical. Estes parâmetros estão representados na Figura 1 (a).

Figura 1: (a) Espaço de busca com o método VLL. (b) Cálculo da similaridade.

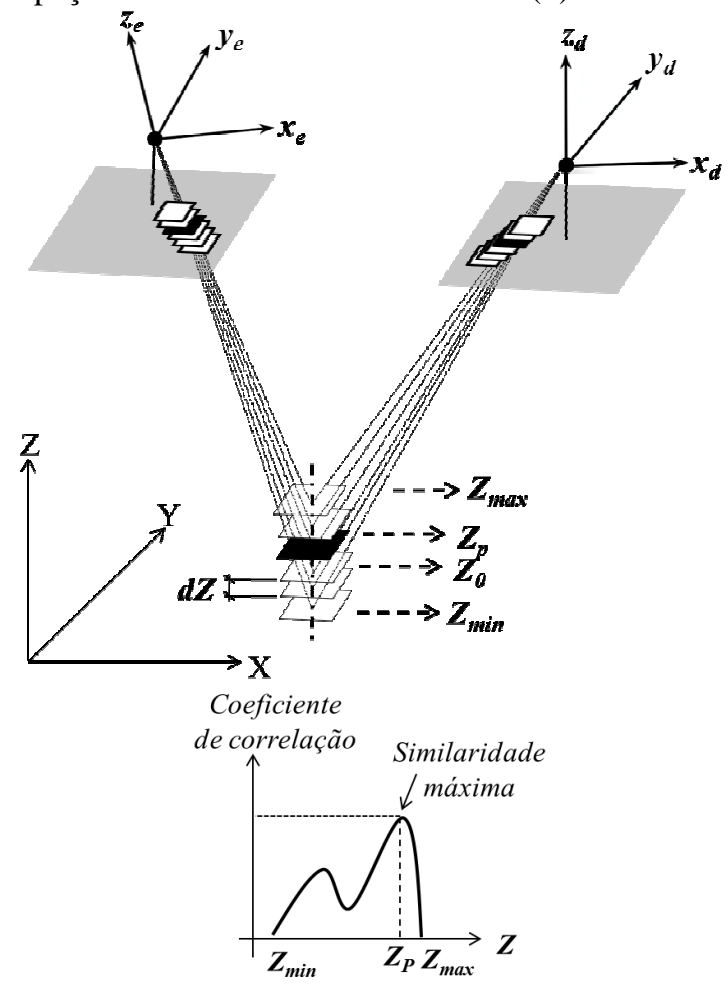

(a)

$\mathrm{O}$ intervalo Zmin e Zmax é dividido em $n$ valores de altitude, onde $n=$ $(\Delta \mathrm{Zmax}+\Delta \mathrm{Zmin}) / d Z$. Com a altitude inicial Zmin e os POE da imagem, calculamse as coordenadas fotogramétricas para o ponto $\mathrm{P}$ em cada imagem em que ele 
apareça, utilizando as equações de colinearidade. Tomando essas coordenadas como centro e uma vizinhança no entorno, são definidas as janelas de referência e de busca para a estimativa da similaridade entre elas. $\mathrm{O}$ valor da altitude é alterado acrescentando-se o incremento $d Z\left(Z_{i}=Z_{0}+i \cdot d Z, \operatorname{com} i\right.$ variando de 1 à $\left.n\right)$, até que o valor Zmax seja alcançado. Repetem-se as etapas de projeção do ponto para as imagens e o cálculo da similaridade. Seleciona-se a altitude $Z_{P}$, no intervalo $Z_{i}$, que apresentar o maior valor de similaridade entre as janelas, como apresentado na Figura 1(b), que mostra o perfil de similaridade ao longo do intervalo de busca $Z_{i}$.

$\mathrm{O}$ método VLL pode ser utilizado com qualquer modelo de sensor e não apenas os de quadro. Pode, ainda, ser utilizado em aplicações de Fotogrametria a curta distância, como mostra Chen et al. (2012). Além disso, possibilita a utilização de mais de duas imagens simultaneamente (PAPARODITIS e POLIDORI, 2002). Isto pode ser realizado verificando-se inicialmente quais imagens contém o ponto do espaço objeto por meio da projeção deste ponto para as imagens. Este ponto é projetado para todas estas imagens e realiza-se o cálculo da similaridade entre as múltiplas janelas delimitadas ao redor do ponto. Como explicado anteriormente para o caso com duas imagens, a altitude $Z_{\min }$ é incrementada por $d Z$ e o processo é repetido até alcançar a altitude máxima do intervalo $\left(Z_{\max }\right)$. Para cada valor de altitude $\left(Z_{i}\right)$ armazena-se um vetor contendo os valores de similaridade entre os pares de janelas das imagens nas quais o ponto aparece. É necessário adotar um critério para análise destas medidas e estimação da altitude que apresenta maior similaridade entre as múltiplas janelas.

Os parâmetros, tamanho do intervalo de busca e incremento, devem ser analisados segundo as características do terreno (montanhoso, plano, urbano com edifícios, etc) e a qualidade dos dados disponíveis e a que se pretende alcançar no MDS. Estes parâmetros influenciam no número de correspondências falsas e corretas e, principalmente, no custo computacional. Uma alternativa para que o VLL seja mais estável e eficiente, é realizar um processo hierárquico, ou seja, a partir de pirâmides de imagens (OLIVEIRA e TOMMASELLI, 2012). Desta forma, o espaçamento $d Z$ é maior no topo da pirâmide (menor resolução) e é reduzido, progressivamente, para os níveis de maior resolução da pirâmide.

\section{GERAÇÃO DE MODELOS DIGITAIS DE SUPERFÍCIE UTILIZANDO IMAGENS ÓPTICAS}

No final do século $\mathrm{XX}$, os softwares comerciais ainda não apresentavam o desempenho exigido em termos de alta qualidade para geração automática de MDS utilizando imagens ópticas (GRUEN, 2012). No entanto, a utilização de câmaras digitais, que melhoraram a dinâmica de manipulação das imagens, em combinação com os avanços nos algoritmos de correspondência, aumentaram as perspectivas de qualidade da aquisição de dados de elevação a partir dos métodos de correspondência de imagens. Haala (2009) chama a atenção para este fato em seu trabalho "Comeback of digital image matching", ressaltando o potencial desta técnica em comparação com a varredura a LASER

Bol. Ciênc. Geod., sec. Artigos, Curitiba, v. 20, no 2, p.257-278, abr-jun, 2014. 
Os algoritmos de correspondência para geração de MDT/MDS podem ser realizados com outros processamentos como a normalização de imagens, quando se realiza a reamostragem epipolar do par de imagens, seguindo-se a determinação da paralaxe dos pontos homólogos e o cálculo da intersecção fotogramétrica para obter as posições tridimensionais destes pontos no espaço objeto (EL-SHEIMY et al., 2005; COSTA et al., 2007). Outras técnicas envolvidas nesses algoritmos são a busca hierárquica; a correspondência incorporando múltiplas primitivas, aplicando os métodos baseados em áreas e baseados em feições (ZHANG, 2005).

A correspondência entre múltiplas imagens possibilita a reconstrução de objetos 3D, combinando-se todas as imagens disponíveis simultaneamente, sem realizar a correspondência de pares de imagens individualmente e posterior fusão dos resultados. É obtida maior precisão na determinação de pontos homólogos pela intersecção de mais de dois feixes de raios da imagem (HAALA, 2011), o que, consequentemente, proporciona a redução dos problemas causados por oclusões, áreas homogêneas e descontinuidades da superfície (GRUEN, 1985; AGOURIS e SCHENK, 1992; ZHANG, 2005; HAALA, 2011). O método VLL pode ser adaptado para realizar a correspondência entre múltiplas imagens e por isso tem sido utilizado em diversos trabalhos com esta finalidade (ZHANG, 2005; SILVEIRA et al., 2007; NOH et al., 2012).

\section{MÉTODO}

O objetivo principal deste trabalho é apresentar uma técnica para a geração de MDS usando a correspondência de múltiplas imagens simultaneamente e o método de busca em linha vertical (VLL), bem como uma avaliação experimental dos resultados obtidos com câmaras digitais de quadro.

O método VLL com múltiplas imagens foi implementado utilizando linguagem de programação $\mathrm{C} / \mathrm{C}++$, com uma sequência a seguir descrita. Antes de iniciar o processo de geração do MDS é necessário definir as coordenadas dos limites da área de interesse para a geração de um MDS aproximado no formato de grade regular. Além disso, devem ser fornecidas as imagens, seus Parâmetros de Orientação Interior (POI), seus POE e os limiares utilizados na correspondência. Em seguida, são geradas as pirâmides de imagens para cada imagem fornecida. A configuração dos parâmetros para a correspondência (tamanho da janela, limiar para o coeficiente de correlação, $\mathrm{d} Z, \Delta \mathrm{Z}_{\mathrm{MAX}}, \Delta \mathrm{Z}_{\mathrm{MIN}}$ ) varia a cada nível da pirâmide, uma vez que cada nível apresenta imagens com resolução diferente. A qualidade dos POE é extremamente importante para o método VLL, pois a correspondência é realizada utilizando a projeção do ponto do espaço objeto para o espaço imagem. Assim, mesmo com a obtenção dos POE por dados de posicionamento por satélite (Global Navigation Satellite System - GNSS) e sistemas inerciais (Inertial Navaigation System - INS) é necessário um "refinamento" destes parâmetros, uma vez que não possuem a acurácia necessária para eliminar totalmente a paralaxe vertical do modelo (CRAMER e STALLMANN, 2001; RUY e TOMMASELLI, 2004). 
Para cada ponto do MDS aproximado, é realizado o processo descrito na Seção 2, considerando a correspondência entre múltiplas imagens e utilizando o coeficiente de correlação, como medida de similaridade.

Os coeficientes de correlação são calculados para cada possível combinação de dois recortes das imagens. A verificação das imagens em que o ponto aparece é feita analisando-se as coordenadas dos cantos das imagens e as coordenadas do ponto. Deste modo, se um ponto está presente em seis imagens, são feitos seis recortes de tamanho pré-determinado, e são calculados quinze coeficientes de correlação entre as combinações de recortes. Estes coeficientes de correlação são comparados a um coeficiente de correlação mínimo aceitável. A média dos valores iguais ou superiores a este limiar é adotada como um coeficiente único, denominado aqui de coeficiente global de correlação. Calcula-se, ainda, o desvio-padrão destes valores. Este procedimento é feito para cada valor de altitude, durante seu incremento.

Figura 2: Processos envolvidos na geração automática do MDS.

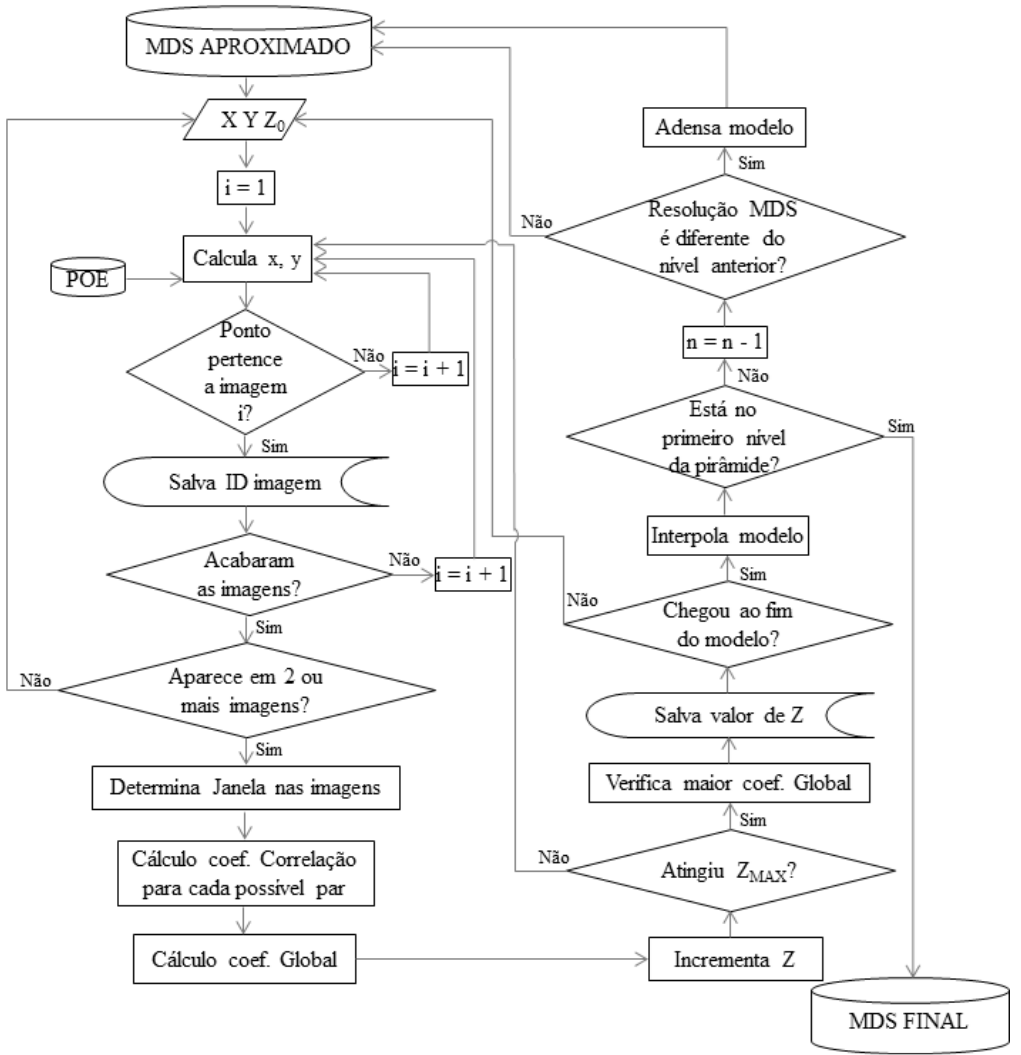

Bol. Ciênc. Geod., sec. Artigos, Curitiba, v. 20, no 2, p.257-278, abr-jun, 2014. 
Ao final, tem-se, para cada valor de $Z_{i}$, um coeficiente global de correlação e seu desvio-padrão; se nenhum coeficiente atingir o limiar de aceitação, o coeficiente global zero é nulo e o ponto recebe altitude zero, valores para os quais assume-se ausência de correspondência. A altitude de um ponto é atribuída verificando-se dentro do intervalo de busca vertical o valor de altitude $Z_{i}$ com o maior coeficiente global e com desvio-padrão menor que o máximo aceitável. O MDS gerado a cada nível da pirâmide é interpolado e utilizado como modelo inicial para o nível subsequente da pirâmide de imagens. As etapas básicas do processo de geração automática do MDS estão representadas no fluxograma da Figura 2.

\section{EXPERIMENTOS E RESULTADOS}

Para os experimentos foram utilizadas imagens obtidas com câmara Hasselblad H4D com 60 megapixels, distância focal calibrada de 50,889 mm. As imagens apresentam um elemento de resolução no terreno (Ground Sample Distance - GSD) de aproximadamente $7 \mathrm{~cm}$. Foram selecionadas seis imagens nas proximidades da FCT-UNESP, Campus de Presidente Prudente, pertencentes a duas faixas do voo (três imagens em cada faixa). Foi realizado o levantamento de pontos de apoio com receptores GNSS de dupla frequência e a fototriangulação das imagens para obtenção dos POE e coordenadas de terreno de pontos fotogramétricos. Os dados de georreferenciamento direto foram utilizados como valores de POE aproximados na fototriangulação.

As imagens foram rotacionadas antes da fototriangulação do bloco, de modo que os referenciais das imagens ficassem com a mesma orientação. Isto foi necessário, pois o programa implementado ainda não considera a variação de orientação entre as faixas no processo de correspondência. Para a geração dos MDSs as imagens rotacionadas foram convertidas para tons de cinza, para diminuir o custo computacional do processamento com os programas desenvolvidos. Os POI foram obtidos no certificado de calibração da câmara fornecido pela empresa Engemap. Na Tabela 1 são apresentados os principais dados das imagens e do voo. A posição do ponto principal obtida do certificado foi alterada de acordo com a rotação realizada nas imagens.

Tabela 1: Dados das imagens e voo.

\begin{tabular}{c|c}
\hline Tamanho do pixel & $6,0 \times 6,0 \mu \mathrm{m}$ \\
\hline $\boldsymbol{x}_{\boldsymbol{0}}$ & $0,00216 \mathrm{~mm} \pm 0,57 \mu \mathrm{m}$ \\
\hline $\boldsymbol{y}_{\boldsymbol{0}}$ & $0,00076 \mathrm{~mm} \pm 0,54 \mu \mathrm{m}$ \\
\hline $\boldsymbol{k}_{\boldsymbol{l}}$ & $-3,37355 \mathrm{e}^{-07} \mathrm{~mm}^{-2} \pm 2,6 \mathrm{e}^{-08} \mathrm{~mm}^{-2}$ \\
\hline $\boldsymbol{k}_{2}$ & $-1,01373 \mathrm{e}^{-09} \mathrm{~mm}^{-4} \pm 5,3 \mathrm{e}^{-11} \mathrm{~mm}^{-4}$ \\
\hline $\boldsymbol{k}_{3}$ & $1,06346 \mathrm{e}^{-12} \mathrm{~mm}^{-6} \pm 3,3 \mathrm{e}^{-14} \mathrm{~mm}^{-6}$ \\
\hline Altitude de voo & $950 \mathrm{~m}$ \\
\hline
\end{tabular}


Para a geração do MDS foram selecionadas pequenas áreas que apresentam diferentes características, que reconhecidamente são complexas para o processo de correspondência entre imagens, como áreas homogêneas, padrão repetitivo, sombras e árvores grandes. Devido à restrição de espaço, neste artigo serão apresentados os experimentos e os resultados de apenas uma das áreas (Quadro 1). Os experimentos e resultados das outras áreas podem ser encontrados em Oliveira (2013). Foram realizados testes alterando as configurações dos parâmetros para a busca de pontos homólogos entre os níveis da pirâmide de imagens e a quantidade de imagens. A superfície topográfica aproximada para entrada no programa foi gerada pela interpolação de todos os pontos envolvidos na fototriangulação (pontos de apoio, de verificação e fotogramétricos) utilizando o interpolador inverso do quadrado da distância e com resolução da grade de $25 \times 25 \mathrm{~cm}$.

Quadro 1: Imagem e descrição da área de estudo.

\begin{tabular}{|c|c|c|}
\hline \multirow{4}{*}{4} & Localização & $\begin{array}{c}\text { Departamento de Cartografia FCT- } \\
\text { UNESP }\end{array}$ \\
\cline { 2 - 3 } & Área & 50x100m \\
\cline { 2 - 3 } & Características & $\begin{array}{c}\text { Áreas homogêneas, árvores grandes } \\
\text { e pequenas, vegetação rasteira, } \\
\text { edificação com telhado com padrão } \\
\text { repetitivo e transparência, veículos, } \\
\text { sombras. }\end{array}$ \\
\cline { 2 - 3 } & $\begin{array}{c}\text { Número de } \\
\text { imagens }\end{array}$ & $\begin{array}{c}\text { 6 (3 imagens na Faixa 1 e 3 } \\
\text { imagens na Faixa 2) }\end{array}$ \\
\hline
\end{tabular}

A verificação da qualidade dos MDSs obtidos pelas técnicas aplicadas foi feita por análise visual e por estatísticas das nuvens de pontos geradas, em comparação com dados de varredura a LASER e com o MDS gerado no módulo Classic ATE do programa LPS da mesma área. Os dados de varredura a LASER foram obtidos no mesmo voo das imagens, utilizando um scanner RIEGL LMS-Q680i, com densidade de aproximadamente 8 pontos $/ \mathrm{m}^{2}$. Com a utilização de pontos de apoio verificou-se que estes dados apresentaram uma tendência, que afetou sua qualidade planimétrica. Este problema ocorreu devido a um erro de sincronismo entre os sensores. Com base nas diferenças entre os pontos de apoio e os pontos da nuvem gerada pelo scanner, foi aplicada uma translação de $4,295 \mathrm{~m}$ na componente $\mathrm{E}$ e de $-0,432 \mathrm{~m}$ na componente $\mathrm{N}$. Os dados utilizados são de apenas uma faixa e nenhum processamento adicional ou filtragem foram realizados na nuvem de pontos, além das translações.

Para a subtração entre as nuvens de pontos foi utilizado o módulo lascontrol do pacote LasTools (ISENBURG, 2013), que gera um TIN utilizando os pontos a 
serem verificados e interpola nele as altitudes correspondentes às posições dos pontos de referência.

A Figura 3 apresenta a distribuição dos pontos de apoio, fotogramétricos e pontos de verificação utilizados na fototriangulação. A Tabela 2 apresenta à média $\mathrm{e}$ a raiz do erro médio quadrático (REMQ) obtidas nos pontos de verificação na fototriangulação realizada no programa de Calibração com Múltiplas Câmaras CMC (BAZAN et al, 2009). Dois pontos, P1 e P4, apresentaram diferenças maiores que 1GSD em uma das componentes $\mathrm{E}$ e $\mathrm{N}$, devido à dificuldades de identificação dos pontos nas imagens. Isto afetou mais a qualidade na componente $\mathrm{N}$, que apresentou REMQ igual a $0,092 \mathrm{~m}$. O resultado foi considerado aceitável para esta aplicação.

Figura 3: Distribuição dos pontos nas imagens.

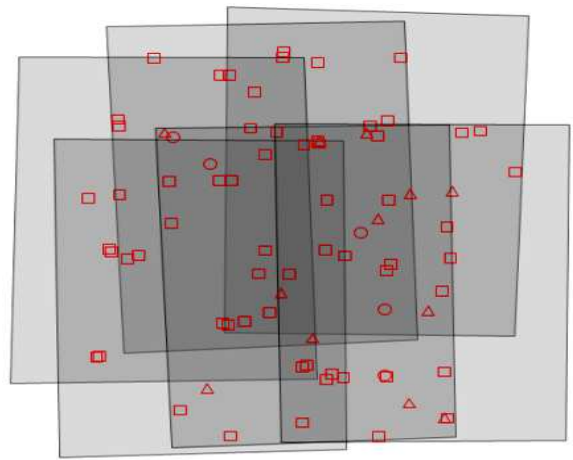

Tabela 2: Discrepâncias, média e REMQ nos pontos de verificação na fototriangulação.

\begin{tabular}{c|c|c|c}
\hline & $\boldsymbol{E}(\boldsymbol{m})$ & $\boldsymbol{N}(\boldsymbol{m})$ & $\boldsymbol{h}(\boldsymbol{m})$ \\
\hline P1 & 0,126 & $-0,038$ & $-0,036$ \\
\hline P2 & 0,049 & 0,074 & $-0,005$ \\
\hline P3 & 0,022 & $-0,098$ & 0,007 \\
\hline P4 & 0,047 & 0,160 & 0,051 \\
\hline P5 & 0,041 & $-0,005$ & 0,114 \\
\hline Média & $\mathbf{0 , 0 5 7}$ & $\mathbf{0 , 0 1 8}$ & $\mathbf{0 , 0 2 6}$ \\
\hline REMQ & $\mathbf{0 , 0 6 7}$ & $\mathbf{0 , 0 9 2}$ & $\mathbf{0 , 0 5 8}$ \\
\hline
\end{tabular}

Foram realizados quatro experimentos (A, B, C e D), a diferença entre eles é a quantidade de imagens utilizadas:

- A: MDS gerado com 6 imagens;

- B: MDS gerado apenas com imagens da Faixa 1 (3 imagens);

- C: MDS gerado apenas com imagens da Faixa 2 (3 imagens);

- D: MDS gerado apenas com um par de imagens. 
Os parâmetros de entrada para geração do MDS são apresentados na Tabela 3. Esta configuração foi adotada para todos os experimentos.

Tabela 3: Configuração dos parâmetros para geração do MDS.

\begin{tabular}{c|c|c|c|c|c}
\hline Nível & $\begin{array}{c}\text { Tamanho da } \\
\text { janela(pixel) }\end{array}$ & $\begin{array}{c}\text { Coefic. de } \\
\text { Correlação Mínimo }\end{array}$ & $\begin{array}{c}\Delta Z \text { max } \\
(\boldsymbol{m})\end{array}$ & $\begin{array}{c}\Delta \text { Zmin } \\
(\boldsymbol{m})\end{array}$ & $\begin{array}{c}\boldsymbol{d Z} \\
(\boldsymbol{m})\end{array}$ \\
\hline 1 & 35 & 0,85 & 20 & 6 & 0,60 \\
\hline 2 & 25 & 0,85 & 7 & 4 & 0,30 \\
\hline 3 & 15 & 0,85 & 3 & 2 & 0,15 \\
\hline 4 & 11 & 0,85 & 1 & 1 & 0,07 \\
\hline
\end{tabular}

Os resultados dos refinamentos do MDS durante o processo hierárquico são apresentados na Figura 4, em forma de imagens em tons de cinza. Os níveis 1, 2, 3 e 4 estão, respectivamente, nas Figuras 4 (a), (b), (c) e (d). O MDS representado na Figura 4 (e) é resultado da aplicação do interpolador inverso do quadrado da distância aos pontos obtidos no nível de maior resolução (Figuras 4 (d)). Nestas imagens os pontos são mais claros quanto maior for sua altitude e quando na cor preta indicam ausência de pontos na região. Como era esperado, tem-se maior definição dos detalhes dos elementos com o aumento da resolução das imagens. Os resultados foram similares para os experimentos B, C e D.

Figura 4: (a), (b), (c) e (d) imagens dos pontos obtidos a cada nível da pirâmide; (e) pontos obtidos após interpolação das altitudes para os pontos sem correspondência, no último nível.

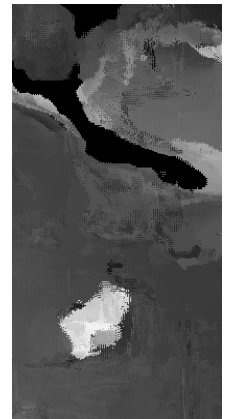

Nível 1

(a)

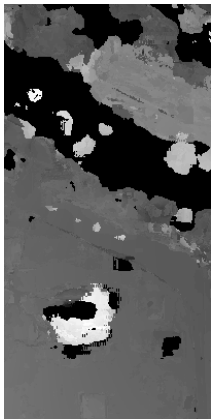

Nível 2

(b)

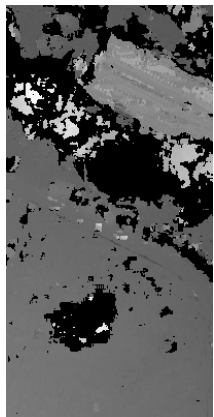

Nível 3

(c)

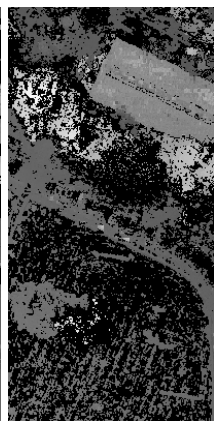

Nível 4

(d)

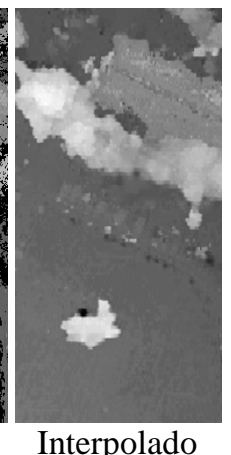

(e)

As imagens dos MDSs gerados em cada experimento são apresentadas na Figura 5 (a), (b), (c) e (d), que correspondem, respectivamente, aos experimentos A, $\mathrm{B}, \mathrm{C}$ e D. 
Oliveira R. A. e Tommaselli, A. M. G.

Figura 5: Imagens hipsométricas dos MDSs gerados (a) experimento A, (b) experimento $\mathrm{B}$, (c) experimento $\mathrm{C}$ e (d) experimento $\mathrm{D}$.

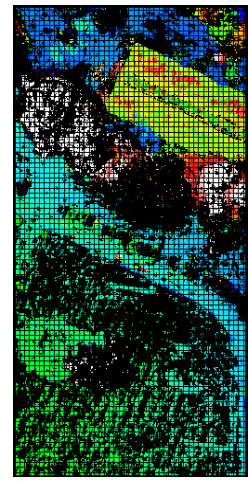

Experimento A

(a)

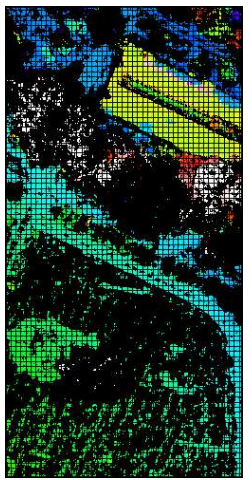

Experimento B

(b)

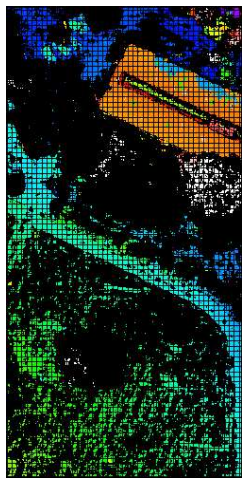

Experimento $\mathrm{C}$

(c)

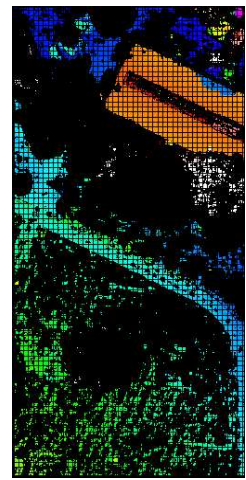

Experimento D

(d)

Figura 6: (a) telhado com padrão repetitivo; (b) telhado obtidos em A; (c) perspectiva isométrica do telhado obtidos em B; (d) vegetação rasteira - área homogênea; (e) vegetação rasteira obtidos em A; (f) vegetação rasteira obtidos em $\mathrm{C}$; (g) árvores de grande porte; (h) árvores obtidos em A; (i) árvores obtidos em D.

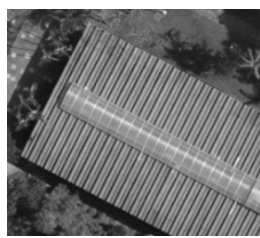

(a)

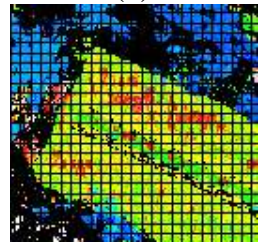

(b)

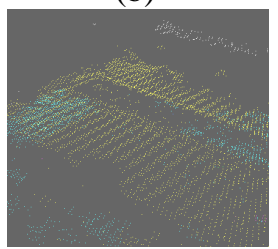

(c)

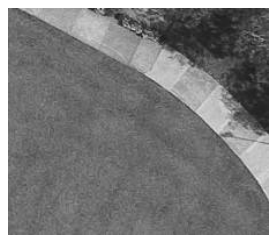

(d)

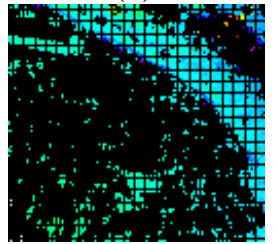

(e)

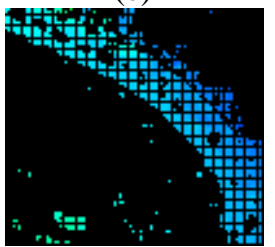

(f)

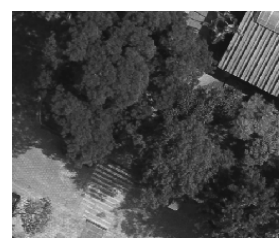

$(\mathrm{g})$

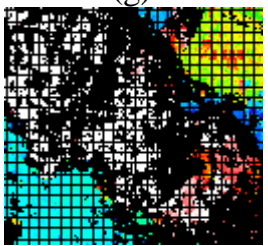

(h)

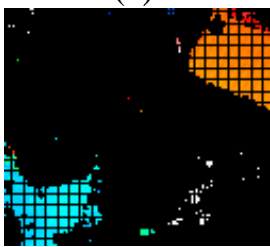

(i)

Bol. Ciênc. Geod., sec. Artigos, Curitiba, v. 20, nº 2, p.257-278, abr-jun, 2014. 
Nota-se visualmente, pela predominância da cor preta, que em todos os experimentos a maior parte dos pontos que não tiveram sua altitude estimada, encontram-se em locais com a presença de árvores ou de vegetação rasteira, que apresenta tonalidade homogênea. Além disso, verifica-se que ocorre uma redução dos pontos determinados corretamente com a diminuição da quantidade de imagens, como pode ser visto ao comparar o MDS representado na Figura 5 (a), em que foram utilizadas seis imagens, com o MDS da Figura 5(d), em que foi usado apenas um par de imagens. Observa-se que a área transparente (centro do telhado Figura 6(a)) também é um elemento difícil para a correspondência de imagens.

Os recortes apresentados na Figura 6 mostram detalhes de algumas áreas específicas dentro do MDS. A edificação com telhado com padrão repetitivo (Figura 6 (a)) teve sua forma delimitada quase completamente, porém, houve maior presença de pontos espúrios no MDS gerado com seis imagens (Experimento A), como mostra a Figura 6 (b). Nos experimentos B, C e D as altitudes dos pontos do telhado apresentaram-se mais uniformes, exceto para alguns pontos próximos às bordas da edificação (Figuras 5 (b), (c) e (d)). O MDS do Experimento B foi o único a recuperar o padrão ondulado do telhado, como mostra a Figura 6 (c).

Para o caso das áreas homogêneas (Figura 6 (d)), a quantidade de pontos estimados foi maior ao utilizar mais imagens, como pode ser visto na Figura 6 (e), que mostra o recorte da imagem hipsométrica do MDS gerado com seis imagens (Experimento A) e na Figura 6 (f), que mostra o recorte do MDS gerado no Experimento $\mathrm{C}$ com três 3 imagens. $\mathrm{O}$ mesmo ocorreu com a presença de árvores (Figuras $6(\mathrm{~g})$ ). O Experimento A estimou mais pontos, como mostra a Figura $6(\mathrm{~h})$, do que o Experimento D (par de imagens), visto na Figura 6 (i).

A comparação da quantidade de pontos do MDS do experimento, com as nuvens de pontos da varredura a LASER e os gerados pelo ATE-LPS, é mostrada na Tabela 4. Entre os experimentos, verifica-se um aumento na quantidade de pontos determinados por correspondência, quando se utiliza a maior quantidade de imagens. Em comparação com o MDS dos dados LASER, a densidade de pontos foi maior nos experimentos utilizando mais de duas imagens. Nota-se ainda que a quantidade de pontos obtida no ATE-LPS foi menor que as obtidas nos demais MDSs dos experimentos realizados com o método proposto.

Tabela 4: Quantidade de pontos obtidos nos experimentos A, B, C e D; por varredura a LASER e no programa ATE-LPS.

\begin{tabular}{c|c}
\hline Fonte dos dados & Quantidade de pontos \\
\hline Experimento A & 37648 \\
\hline Experimento B & 27413 \\
\hline Experimento C & 24947 \\
\hline Experimento D & 21073 \\
\hline LASER & 24616 \\
\hline ATE-LPS & 7985 \\
\hline
\end{tabular}


A Tabela 5 mostra a média, o desvio-padrão e a REMQ obtidos da subtração entre os dados LASER e os MDSs gerados pelo método VLL, sem e com interpolação após o final da estimação das altitudes dos pontos.

Tabela 5: Média, desvio-padrão e a REMQ das diferenças entre os dados LASER e os MDSs gerados.

\begin{tabular}{c|c|c|c|c|c|c|c|c}
\hline & \multicolumn{2}{|c|}{$\boldsymbol{A}$} & \multicolumn{2}{c|}{$\boldsymbol{B}$} & \multicolumn{2}{c|}{$\boldsymbol{C}$} & \multicolumn{2}{c}{$\boldsymbol{D}$} \\
\hline & $\begin{array}{c}\text { Sem } \\
\text { interp. }\end{array}$ & Interp. & $\begin{array}{c}\text { Sem } \\
\text { interp. }\end{array}$ & Interp. & $\begin{array}{c}\text { Sem } \\
\text { interp. }\end{array}$ & Interp. & $\begin{array}{c}\text { Sem } \\
\text { interp. }\end{array}$ & Interp. \\
\hline Média $(\boldsymbol{m})$ & 0,114 & $-0,130$ & 0,053 & $-0,254$ & $-0,349$ & $-0,725$ & $-0,223$ & $-0,679$ \\
\hline $\boldsymbol{\sigma}(\boldsymbol{m})$ & 2,997 & 3,093 & 3,019 & 3,145 & 3,054 & 3,250 & 2,835 & 3,075 \\
\hline $\boldsymbol{R E M Q}(\boldsymbol{m})$ & 3,000 & 3,096 & 3,019 & 3,155 & 3,074 & 3,330 & 2,844 & 3,149 \\
\hline
\end{tabular}

Os resultados da Tabela 5 mostram que em todos os experimentos as REMQs ficaram em torno de três metros, o que indica um conjunto de pontos com erros de grande magnitude, em comparação com o GSD da imagem. Para separar os conjuntos de pontos de acordo com a magnitude das discrepâncias entre o MDS LASER e o MDS VLL foram gerados histogramas de frequência separados em sete intervalos, variando de 0,0-0,1 $\mathrm{m}$ a maior que $0,7 \mathrm{~m}$. Na Figura 7 são apresentados histogramas que mostram a porcentagem dos valores absolutos das diferenças entres os pontos LASER e os pontos de cada experimento, antes e após a interpolação dos pontos que não foram gerados por correspondência.

Os gráficos da Figura 7 mostram que, em todos os experimentos, aproximadamente 35\% das discrepâncias entre os MDSs obtidos e os dados LASER foram maiores que $0,5 \mathrm{~m}$ e cerca de $55 \%$ foram menores que $0,3 \mathrm{~m}$. Apenas os experimentos A e B apresentaram mais de $40 \%$ dos pontos com diferenças de até $0,2 \mathrm{~m}$. Em todos os histogramas o maior percentual corresponde a pontos com diferenças maiores que $0,7 \mathrm{~m}$, o que explica os valores altos das REMQs apresentadas na Tabela 5. Para explicar estas diferenças foi produzido um gráfico

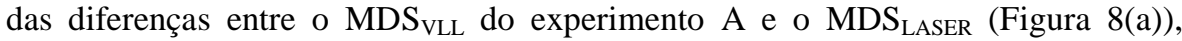
correspondente a um recorte da imagem aérea (Figura 8(b)). A análise visual permite verificar que as maiores discrepâncias estão relacionadas, sobretudo, aos pontos nas áreas com árvores (áreas destacadas e assinaladas com (a) e (b) na Figura 8), que é um dos elementos mais complexos para a correspondência de imagens, devido às suas características geométricas e na área transparente no telhado da edificação (assinalado com (c) na Figura 8). Além disso, os dados LASER possuem pontos que ultrapassaram elementos como as folhas das árvores, o que não ocorre ao utilizar o processo de correspondência com imagens ópticas. Com isso, a diferença entre estes pontos pode aproximar-se do valor da altura das árvores, como se observa na Figura 8. É válido ressaltar que não foi realizada a filtragem nos MDSs gerados após a correspondência, ou seja, a quantidade de pontos espúrios não foi reduzida. A diferença entre os dados sem interpolação e após a interpolação é 
devida à uma suavização dos dados e geração de novos pontos, nos quais a altitude não havia sido estimada por correspondência.

Figura 7: Histogramas da porcentagem das discrepâncias.

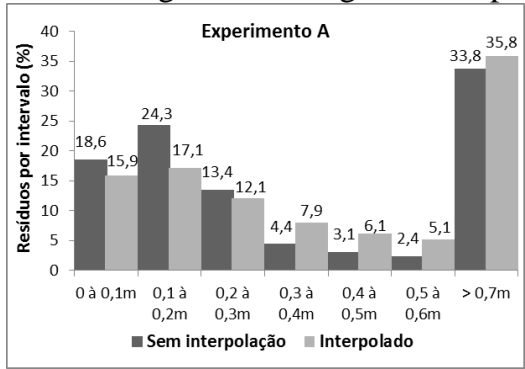

(a)

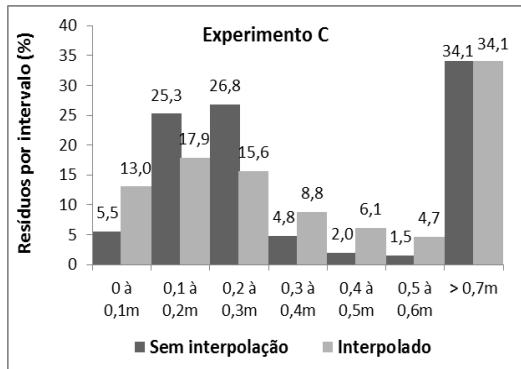

(c)

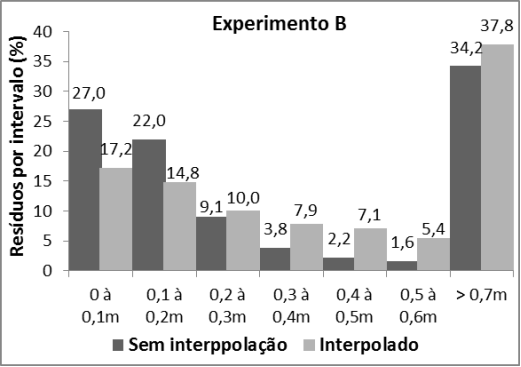

(b)

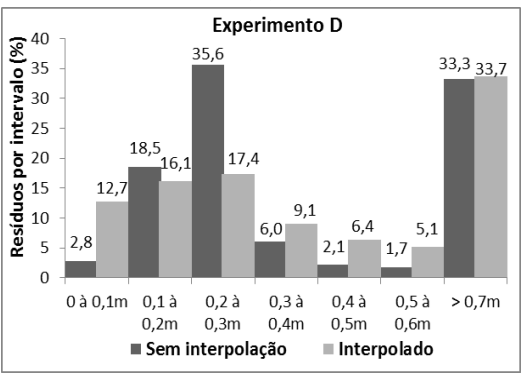

(d)

Figura 8: (a) Vista perspectiva das diferenças entre o $\mathrm{MDS}_{\mathrm{VLL}}$ do Experimento A e os dados LASER. (b) Recorte de uma imagem aérea da área correspondente.

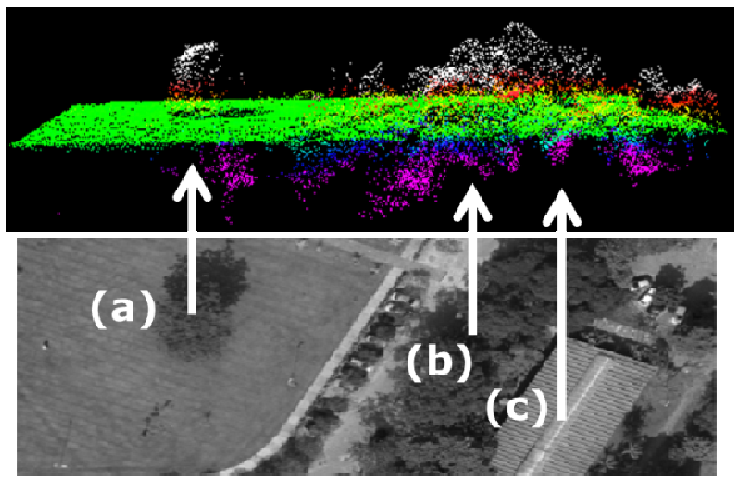

(a)

(b)

Para a comparação com dados gerados também por um método fotogramétrico tradicional, no qual são utilizados pares de imagens, foi gerado um MDS no 
software comercial LPS, em seu módulo Classic ATE. Este módulo não realiza a correspondência entre múltiplas imagens, mas gera um MDS para cada estereopar. A Figura 9 mostra a imagem hipsométrica do $\mathrm{MDS}_{\mathrm{LPS}}$ obtido para um par de imagens.

Figura 9: Imagem hipsométrica do MDS gerado no ATE-LPS.

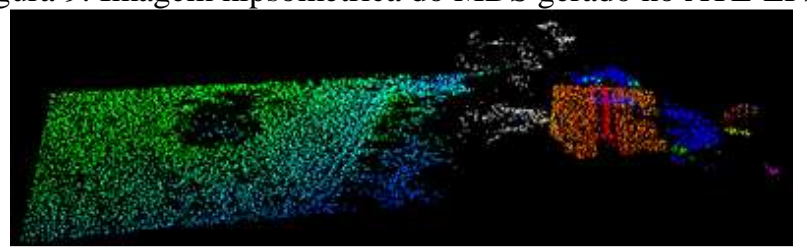

Nota-se pela análise visual do $\mathrm{MDS}_{\text {ATE-LPS, }}$ que os maiores problemas para a obtenção de altitudes ocorreram em locais com árvores e no telhado com padrão repetitivo, assim como ocorreu no Experimento D (Figura 4 (d)), que utilizou apenas um par de imagens. Pela Tabela 4 verifica-se que a densidade de pontos é inferior à densidade obtida nos experimentos utilizando múltiplas imagens simultaneamente. A obtenção de mais pontos, principalmente nas áreas problemáticas para o processo de determinação automática de pontos homólogos, permite inferir que a utilização de múltiplas imagens simultaneamente e não apenas pares, reduz os problemas como oclusões e áreas homogêneas.

Figura 10: Histogramas das discrepâncias $\left(\mathrm{MDS}_{\mathrm{LASER}}-\mathrm{MDS}_{\mathrm{ATE}-\mathrm{LPS}}\right)$ por intervalos.

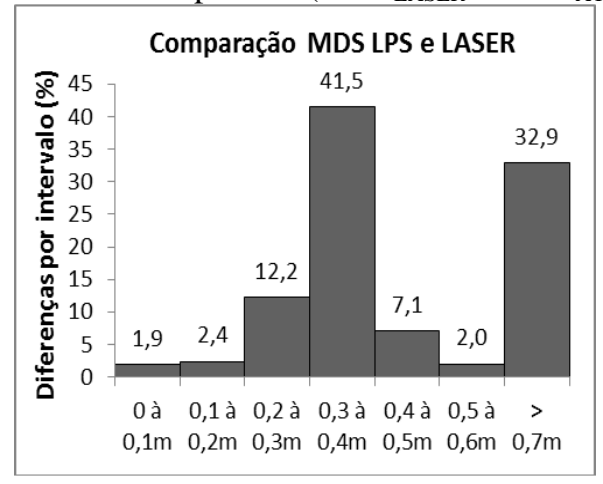

A subtração entre os $\mathrm{MDSs}_{\mathrm{VLL}}$ obtidos nos experimentos A, B, C e D e o $\mathrm{MDS}_{\text {ATE-LPS }}$ gerado no ATE-LPS resultou nas médias, desvios-padrão e nas REMQs das diferenças apresentadas na Tabela 6. Os histogramas com a porcentagem das diferenças $\left(\mathrm{MDS}_{\mathrm{ATE}-\mathrm{LPS}}-\mathrm{MDS}_{\mathrm{VLL}}\right)$ por intervalo são apresentados na Figura 11. 
Tabela 6: Média, desvio-padrão e a REMQ das discrepâncias entre o $\operatorname{MDS}_{\text {ATE-LPS }}$ e os MDSs $s_{\text {VLL }}$.

\begin{tabular}{c|c|c|c|c|c|c|c|c}
\hline & \multicolumn{2}{|c|}{$\boldsymbol{A}$} & \multicolumn{2}{c|}{$\boldsymbol{B}$} & \multicolumn{2}{c|}{$\boldsymbol{C}$} & \multicolumn{2}{c}{$\boldsymbol{D}$} \\
\hline & $\begin{array}{c}\text { Sem } \\
\text { interp. }\end{array}$ & Interp. & $\begin{array}{c}\text { Sem } \\
\text { interp. }\end{array}$ & Interp. & $\begin{array}{c}\text { Sem } \\
\text { interp. }\end{array}$ & Interp.. & $\begin{array}{c}\text { Sem } \\
\text { interp. }\end{array}$ & Interp. \\
\hline Média $(\boldsymbol{m})$ & $-0,127$ & $-0,307$ & $-0,201$ & $-0,457$ & $-0,235$ & $-0,479$ & $-0,164$ & $-0,444$ \\
\hline $\boldsymbol{\sigma}(\boldsymbol{m})$ & 1,145 & 1,227 & 1,240 & 1,344 & 1,133 & 1,224 & 1,175 & 1,246 \\
\hline $\boldsymbol{R E M Q}(\boldsymbol{m})$ & 1,152 & 1,264 & 1,256 & 1,419 & 1,157 & 1,314 & 1,186 & 1,313 \\
\hline
\end{tabular}

Figura 11: Histograma da porcentagem das discrepâncias $\left(\mathrm{MDS}_{\mathrm{ATE}-\mathrm{LPS}}-\mathrm{MDS}_{\mathrm{VLL}}\right)$ por intervalos.

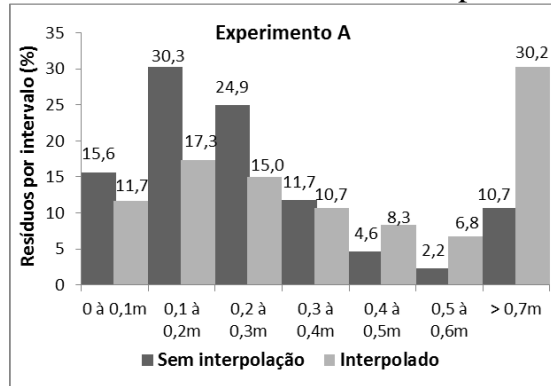

(a)

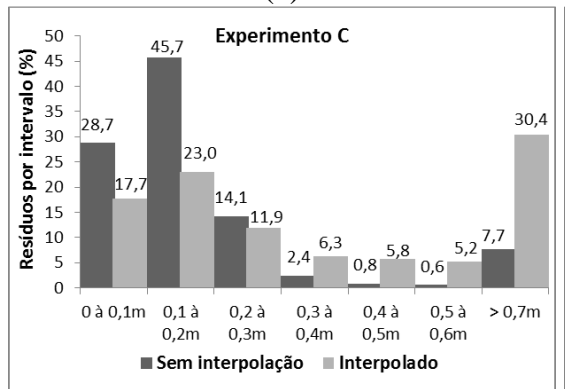

(c)

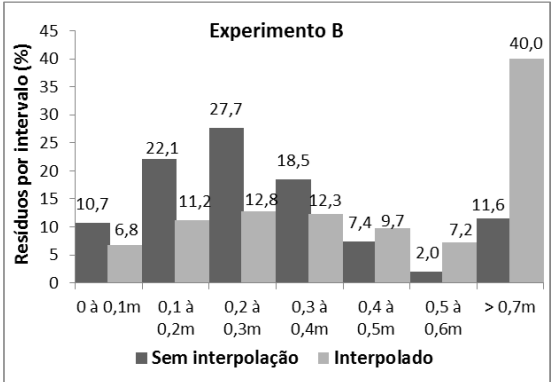

(b)

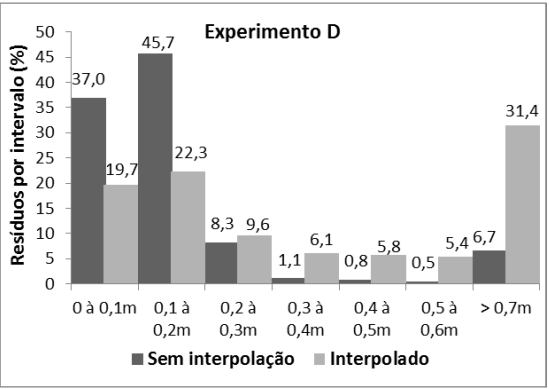

(d)

Os histogramas da Figura 11 mostram que no Experimento A 12,9\% das discrepâncias foram maiores do que $0,5 \mathrm{~m}$ e $45,9 \%$ foram menores que $0,2 \mathrm{~m}$ (Figura 11(a)). No experimento B (Figura 11(b)), 12,6\% dos pontos apresentaram discrepâncias maiores do que $0,5 \mathrm{~m}$ e $32,8 \%$ menores que $0,2 \mathrm{~m}$. Ao contrário da verificação com dados LASER, os experimentos C (Figura 11(c)) e D (Figura 11(d)) obtiveram menores diferenças, principalmente no caso $\mathrm{D}$, onde apenas $7,1 \%$ foram maiores que $0,5 \mathrm{~m}$ e $82,7 \%$ menores que $0,2 \mathrm{~m}$.

A alteração nos histogramas entre os diferentes dados comparados está relacionada, principalmente, à diferença na densidade de pontos obtida em áreas de 
vegetação, que foi menor nos experimentos $\mathrm{C}$ e $\mathrm{D}$, porém similares à obtida no ATE-LPS. A maior quantidade de pontos gerados, principalmente, nas áreas com a presença de árvores e em áreas homogêneas, é uma vantagem importante da utilização da correspondência entre múltiplas imagens.

\section{CONCLUSÕES}

Neste trabalho, uma metodologia para a geração de MDSs utilizando o método de busca em linhas verticais (VLL) adaptado para a utilização de múltiplas imagens foi implementada e avaliada experimentalmente. Foram realizados experimentos usando uma área com diferentes características, que dificultam o procedimento de correspondência de imagens, como árvores, padrão repetitivo e área homogênea.

A análise visual dos MDSs gerados mostrou que a quantidade de pontos gerados por correspondência em áreas homogêneas e, principalmente, em árvores foi maior com o aumento da quantidade de imagens. No telhado com padrão repetitivo, os pontos gerados foram mais fiéis à sua forma original, ao considerar a correspondência entre as faixas separadamente, o que deve estar relacionado às variações geométricas devidas à diferença de orientação entre as faixas, que não é considerada na função de similaridade utilizada. A comparação entre o MDS obtido no software ATE-LPS e os dados de varredura a LASER, apresentou desvio-padrão e REMQ similares aos obtidos com a metodologia proposta neste trabalho. De uma forma geral, os experimentos utilizando múltiplas imagens proporcionaram maior qualidade e densidade de pontos. No entanto, a grande quantidade de pontos gerados também acarretou em maior número de pontos espúrios. A qualidade dos resultados obtidos foi melhor em áreas com pavimento e locais com vegetação rasteira (áreas homogêneas). As áreas que apresentaram maior dificuldade para a estimação das altitudes por correspondência foram aquelas com árvores de grande porte.

Um aspecto que afetou significativamente a análise da qualidade da comparação está relacionado à qualidade dos dados LASER utilizados neste experimento. As translações aplicadas não são suficientes para corrigir totalmente os efeitos do erro de sincronismo. Além disso, os dados de varredura a LASER possuem qualidade planimétrica inferior à obtida nos MDSs utilizando imagens ópticas.

Os resultados obtidos neste trabalho mostraram que, ao utilizar múltiplas imagens simultaneamente no processo de correspondência, os problemas de correspondência em áreas homogêneas, vegetação e padrão repetitivo são reduzidos, devido à a maior possibilidade de estimação de pontos nestas áreas críticas. Verificou-se experimentalmente que as imagens ópticas permitem a geração de MDS com densidade e qualidade comparáveis aos gerados por varredura à LASER, com algumas exceções discutidas no trabalho.

No trabalho realizado não foram implementadas algumas técnicas como o refinamento da correspondência pelo método dos mínimos quadrados (MMQ), que permite a estimação da correspondência com precisão subpixel e consideração das variações geométricas. Além disso, pode-se implementar métodos de filtragem do 
MDS para redução de pontos espúrios e utilizar funções de correspondência para considerar as informações de todas as bandas das imagems(SILVA et al., 2007). As áreas que representam um desafio para o problema de correspondência de imagens, como áreas homogêneas, padrões repetitivos e árvores, merecem mais estudos. Estes tópicos são sugeridos para trabalhos futuros com potencial de melhoria dos resultados apresentados. Tendo em vista todos os procedimentos implementados e os métodos descritos para a geração de MDS propostos neste trabalho, ressalta-se que o tempo de processamento é grande, devido ao alto custo computacional. Com isso, sugere-se, ainda, o estudo de métodos de otimização para redução do tempo de processamento, como o uso de processamento paralelo e processamento integrado com as placas gráficas.

\section{AGRADECIMENTOS}

Os autores agradecem à FAPESP (Fundação de Amparo à Pesquisa do Estado de São Paulo) pelo apoio concedido por meio de uma bolsa de mestrado (processo: 2010/14444-0).

\section{REFERÊNCIAS BIBLIOGRÁFICAS}

AGOURIS, P.; SCHENK, T. Multiple image matching. In: ISPRS, 1992, Washington. International Archives of Photogrammetry and Remote Sensing. Washington: ISPRS, 1992 v. XXIX, Parte B3, p. 802-807.

BAZAN, W. S., TOMMASELLI, A. M. G, GALO, M., RUY, R. S. Influência das injunções de orientação na calibração de um sistema dual de câmaras digitais. Boletim de Ciências Geodésicas. , v.15, p.444 - 466, 2009.

CHEN, Y.; TSENG, Y.; HSIEH, C. WANG, P.; TSAI, P. Object-space multi-image matching of mobile-mapping-system image sequences. In: XXII Congress of the ISPRS, 2012, Melbourne. International Archives of the Photogrammetry, Remote Sensing and Spatial Information Sciences, 2012. v. XXXIX. p. 465470.

COSTA, E. R.; TOMMASELLI, A. M. G.; GALO, M. Geração automática de modelos digitais de terreno a partir de imagens obtidas por câmaras digitais. Revista Brasileira de Cartografia, Rio de Janeiro, R.J, 2007, v. 59, n. 3, p. 151170.

CRAMER, M.; STALLMANN, D. On the use of GPS/inertial Exterior Orientation Parameters in Airborne Photogrammetry. In: OEEPE Workshop on Integrated sensor orientation, 2001, Hannover. Proceedings... Hannover: OEEPE, 2001. p. 32-44.

EL-SHEIMY, N.; VALEO, C.; HABIB, A. Digital terrain modeling: acquisition, manipulation, and applications. Artech House, 2005. 257p.

GRUEN, A.W. Adaptive least squares correlation: A powerful image matching technique. Journal of Photogrammetry, Remote Sensing and Cartography. p.175-187, 1985. 
GRUEN, A. Development and status of image matching in photogrammetry. The Photogrammetric Record, v.27, n. 137, p. 36-57, 2012.

HAALA, N. Comeback of Digital Image Matching. In Photogrammetric Week, 2009, Heidelberg, 2009. Proceedings... Heidelberg: Ed. D. Fritsch, 2009. p. 289-301.

HAALA, N. Multiray Photogrammetry and Dense Image Matching. In Photogrammetric Week, Heidelberg, 2011. Proceedings.... Heidelberg: Ed. D. Fritsch, 2011. p. 185-195.

HEIPKE, C. Overview of image matching techniques. In: Workshop on the Application of digital photogrammetric workstations, 1996, Lausanne. Proceedings... Lausanne: OEEPE, 1996.

IDOETA, I. V. Metodologia de elaboração automatizada de modelo digital de elevação e ortofoto em média e pequena escala. 2007. 215 f. Tese (Doutorado em Engenharia) Escola Politécnica da Universidade de São Paulo, São Paulo, 2007.

ISENBURG, M. LASTOOLS: Software for rapid converting, filtering, viewing, gridding, and compressing of LiDAR. Disponível em: <http://www.cs.unc.edu/ isenburg/lastools/>. Acesso em 11 março 2013.

NOH, M.; CHO, W.; BANG, K. Highly Dense 3D Surface Generation Using Multiimage Matching. ETRI Journal, v. 34, n. 1, p. 87-97, 2012.

OLIVEIRA, R. A.; TOMMASELLI, A. M. G. Geração automática de Modelos Digital de Superfície utilizando múltiplas imagens. In: III Simpósio Brasileiro de Geomática, 2012, Presidente Prudente. Anais... Presidente Prudente: III SGB, 2012. v. 1. p. 193-198.

OLIVEIRA, R. A. Geração de Modelos Digital de Superfície utilizando múltiplas imagens. 2013. 133f. Dissertação (Mestrado em Ciências Cartográficas) Faculdade de Ciências e Tecnologia, Universidade Estadual Paulista, Presidente Prudente.

PAPARODITIS, N.; POLIDORI, L. DSM quality: internal and external validation. In: EGELS, Y.; KASSER, M. Digital Photogrammetry. 1. ed. New York: Taylor \& Francis, 2002. 351p.

ROTHERMEL, M.; HAALA, N. Potential of Dense Matching for the Generation of High Quality Digital Elevation models. In: ISPRS Workshop High-Resoultion Earth Imaging for Geospatial Information, 2011, Proceedings... Hannover: ISPRS, 2011.

RUY, R.S.; TOMMASELLI, A. M. G. Reconstrução automática de estereomodelos a partir de parâmetros de orientação direta do sensor. Boletim de Ciências Geodésicas, Curitiba, PR, v. 10, n. 1, p. 65-82, 2004.

SCHENK, T. Digital Photogrammetry. v.I. Ohio: Terra Science, 1999. 428 p.

SILVA, P. H. A.; TOMMASELLI, A. M. G.; GALO, M. Um estudo sobre o uso do modelo de cor RGB no processo de correspondência por correlação. Boletim de Ciências Geodésicas, v.13, n.1, 2007. 
SILVEIRA, M. T; NUNES, J. L. S. B.; MOTA, G. L. A.; BERNARDO FILHO, O.; RIBEIRO, J. A.; CAOLHO FILHO, L. C. T.; SILVEIRA, F. J. C.; SANTOS, F. C. Geração de Modelo Digital de Elevações e orto-retificação: a experiência do projeto E-FOTO. In: XXIII Congresso Brasileiro de Cartografia, 2007, Rio de Janeiro. Anais: XXIII CBCG, 2007, p. 832-839.

ZHANG, L. Automatic Digital Surface Model (DSM) Generation from Linear Array Images. 2005. Ph. D. Dissertation, n. 88, Institute of Geodesy and Photogrammetry, ETH Zurich, Switzerland. Díponível em <http://ecollection.ethbib.ethz. ch/ecol-pool/diss/fulltext/eth16078.pdf $>$.Acesso em 6 de março de 2013.

(Recebido em abril de 2013. Aceito em fevereiro de 2014). 